\title{
An exploration of lexical selection in adults who stutter
}

\author{
Amanda S. Lee ${ }^{\mathrm{a}}$, Greg A. O'Beirne ${ }^{\mathrm{a}}$ and Michael P. Robb ${ }^{\mathrm{b}, *}$ \\ ${ }^{\mathrm{a}}$ School of Psychology, Speech and Hearing, University of Canterbury, Christchurch, New Zealand \\ ${ }^{\mathrm{b}}$ School of Health Sciences, University of Canterbury, Christchurch, New Zealand
}

Received 22 May 2021

Accepted 3 August 2021

\begin{abstract}
.
BACKGROUND AND OBJECTIVE: People who stutter (PWS) are able to anticipate a moment of stuttering. We wished to explore whether this anticipation might be reflected by either unusual word choice and/or delayed word production during a single-word confrontation naming task.

METHOD: Nine PWS and nine age- and sex-matched fluent controls completed the single-word confrontation-naming task. Groups were compared on numbers of word-finding and fluency errors, response latency, and naming accuracy, measured against a novel 'usuality' criterion. Regression modelling of response accuracy and latency was conducted.

RESULTS: The groups did not differ on naming task performance, except for a greater frequency of response latency errors in the PWS group. For both groups, responses containing word-finding or fluency errors were more likely to be non-usual names, and these were associated with longer latencies than accurate responses. For PWS, latency was positively related to participant age, and accuracy inversely related to stuttering severity.

CONCLUSIONS: The findings provide insights into word substitution as a generalized behaviour, its function, and associated time-cost. Group-specific relationships imply greater sensitivity in PWS to changing demands and capacities, and highlight the complexity of interactions between physical stuttering behaviour and verbal avoidance.
\end{abstract}

Keywords: Stuttering, verbal avoidance, word substitution, word choice, picture naming, response latency

\section{Introduction}

Briley and Kalinowski (2016) suggest that stuttering is an experiential disorder, where the person who stutters (PWS) continually thinks about stuttering. The PWS does not need to actually stutter to experience stuttering (Tichenor \& Yaruss, 2019). The PWS is able to anticipate a moment of stuttering, and this anticipation effect is a core feature of several theories of stuttering (Garcia-Barera \& Davidow, 2015). The impact of stuttering anticipation on communication

\footnotetext{
${ }^{*}$ Corresponding author: Michael P. Robb, University of Canterbury, Private Bag 4800, Christchurch 8140, New Zealand. E-mail: michael.robb@canterbury.ac.nz.
}

is not limited to speech disfluency, but also implicates reduced flexibility of language construction (Lee et al., 2016a, 2016b; 2015) and verbal avoidance (Iverach et al., 2011; Jackson et al., 2015; Plexico et al., 2009).

\subsection{Avoiding words}

Decades ago, Petrunik and Shearing (1983) stated that verbal avoidance represents the PWS attempts to conceal their stuttering. Avoidance behaviour takes various forms in conversational speech and may include word substitution or omission, circumlocution, and simply 'letting others talk', as a means of protection from the emotional harm of stuttering and 
to maintain the identity of a normally fluent speaker (Cream et al., 2003; Guitar, 2019; Lowe et al., 2017; 2021; Plexico et al., 2009). When a person avoids words, s/he either needs to choose a different word or change what is to be said (Lowe et al., 2017). As a result, behavioural reinforcement occurs, giving rise to habitual patterns of speech avoidance in PWS. Although there is wide variation in the form and frequency of avoidance strategies used by PWS (Crichton-Smith, 2002; Mancinelli, 2018; Plexico et al., 2009), data suggest that word substitution is one of the most commonly employed and earliestappearing strategies (Crichton-Smith, 2002; Jackson et al., 2015), accounting for up to $82 \%$ of all coping responses (Vanryckeghem et al., 2004).

Successfully 'problem-solving' anticipated stuttering through word substitution and other similar strategies has been reported to result in distraction, miscommunication, restricted opportunities, and reduced capacity to communicate for social purposes (Amster \& Klein, 2018; Cream et al., 2003; Jackson et al., 2015; Plexico et al., 2009). Indeed, linguistic data concerning the expression of politeness and opinion by PWS suggest a reduced openness to interpersonal engagement in these speakers, which the researchers interpret as evidence of systemic language change related to verbal avoidance (Lee et al., 2015). Post-treatment interviews with PWS indicate the persistence of avoidance behaviours in some cases, where a cumulative load of typical speech content planning, anticipatory word selection, and the application of newly learned stutter-control techniques then becomes apparent (Cream et al., 2003; Cream et al., 2004; Linklater, 2020).

Verbal avoidance is a significant feature in the clinical presentation of stuttering, with far-reaching implications for the communicative freedom and effectiveness of PWS. To date, the majority of research examining verbal avoidance in PWS is based on personal accounts during structured or semistructured interviews (Cream et al., 2003, 2004; Crichton-Smith, 2002; Jackson et al., 2015; Plexico et al., 2009) and self-report checklists (Blomgren et al., 2005; Vanryckeghem et al., 2004). That is, the only essential evidence of an instance of spontaneous verbal avoidance resides in the speaker's own awareness. The aim of the current study was to explore the use of a behavioural measure to examine verbal avoidance. Creating a measurable context for objectively documenting avoidance behaviour could provide additional insight regarding the experience of stuttering. Research related to confrontational picture naming performance in PWS may indicate one possible approach.

\subsection{Clues from confrontation naming}

Lexical access is a highly automated process but not entirely passive, as the speaker exerts some degree of cognitive control over the process. According to Miyake et al. (2000) and others (Levelt et al., 1999; Shao et al., 2013; Shao et al., 2014), naming pictures involves executive control over lexical competitors. That is, word selection is achieved by suppressing the activation of alternative lexical choices. Presumably, the speed at which a picture is named (i.e., latency) is representative of the amount of competition among possible word choices (Folstein \& Van Petten, 2008). A picture that could be named using a variety of word choices would result in a longer latency of response.

Interest in the relationship between lexical processing ability and stuttering has given rise to a cluster of studies examining the performance of PWS on tasks requiring the rapid naming of picture stimuli. These have attempted to explain disfluency in relation to models of lexical access, activation, and production, using both behavioural (e.g., Crowe \& Kroll, 1991; Hennessey et al., 2008; Newman \& Bernstein Ratner, 2007; Pellowski, 2011; Prins et al., 1997; Taylor et al., 1970) and electrophysiological data (e.g., Maxfield, 2020, 2021; Maxfield et al., 2010; Maxfield et al., 2015). Results from confrontation naming tasks in PWS have been equivocal, producing a complex picture of lexical access behaviour in PWS. For example, Maxfield et al. (2015) and Pellowski (2011) reported on groups of PWS and people with no stuttering (PWNS) who completed computerized naming tasks, where the target responses were noun labels for common objects. Both studies found a significantly longer naming latency for PWS participants, which was interpreted to reflect diminished ability to control lexical selection. However, similar studies reporting no significant naming latency differences between PWS and PWNS also exist (Hennessey et al., 2008; Maxfield, 2020, 2021; Newman \& Bernstein Ratner, 2007, Prins et al., 1997). Prins et al. found no significant main effect of group status on the speech onset latency of 12 PWS and 12 PWNS, in response to line drawings depicting target nouns or verbs. A comparatively greater difference in response latency was nonetheless seen between groups for target verbs than nouns. Based on this observation and the tendency for disfluency to appear on verb phrases from the time of early childhood 
(Bloodstein et al., 2021), the authors suggested a particular role of slowed verb processing in precipitating stuttering. In the case of Maxfield (2020, 2021), the latency of naming words depicting common and uncommon objects/actions was measured in PWS and PWNS. Both groups were found to exhibit differential latencies for common (short) and uncommon (long) words. However, the magnitude of the effect was larger for PWS.

There is evidence that, when compared to PWNS on speech latency measures, PWS respond differently to changes in processing demands, such as those related to linguistic complexity and emotionality (Hennessey et al., 2014; Newman \& Bernstein Ratner, 2007; Prins et al., 1997; Tsiamtsiouris \& Cairns, 2009). While confrontation naming thus far has been used to examine lexical access and activation among PWS, the methodology also allows for the accuracy of naming responses. Errors in naming have typically included any verbal responses other than a pre-determined target word, as well as hesitations, stutters, and non-responses, and have not been shown to distinguish groups of PWS and PWNS (e.g., Hennessey et al., 2008; Maxfield et al., 2010, 2015; Pellowski, 2011). Newman and Bernstein Ratner (2007) employed a more elaborate method of accuracy analysis, with interesting results. The study involved a timed computerized picture-naming task containing noun and verb stimuli controlled for word frequency factors and matched for initial phoneme, which was completed by 25 PWS and 25 PWNS participants. Response errors resulting from wordfinding difficulty or visual confusion of the pictured stimulus were tallied, but response variants representing dialectal differences, alternate word choices, or elaborations, were counted separately and classified as correct. This was intended to account for any occurrences of word avoidance. Statistical analysis revealed that the PWS group produced a significantly higher frequency of errors than the control group, but the two groups demonstrated a similar frequency of response variants and elaborations. Notably, the authors also described the use of certain highly unusual word labels by PWS participants to name common picture stimuli. For example, boy was labelled androgyne, and knee was termed patella. Though the incidence of such responses was low, and the overall number of variant responses was not significantly different between groups, the authors postulated that such lexically creative word choices might reflect word substitution for stuttering avoidance. In the present study, we wished to explore the possibility of capturing instances of stuttering avoidance on the basis of word choice.

Newman and Bernstein Ratner's (2007) findings invite the closer evaluation of confrontation naming response variants, which, if qualified in relation to a criterion, could provide insights into word selection behaviour (rather than simply lexical processing ability) in PWS. These data could be combined with naming latency measurements to examine the timecost associated with problem-solving stuttering "in the moment." Admittedly, the confrontation naming paradigm represents a contrived speaking context, whereas verbal avoidance is a feature of spontaneous conversation. Yet, the particular salience of word substitution as an avoidance strategy among PWS provides certain grounds for taking this approach, with its word-production focus, as an exploratory point of departure.

\subsection{The present study: picture naming task}

Evidence from self-report sources demonstrates that verbal avoidance, of which word substitution is a key strategy, can significantly impact the quality of communication experienced by PWS (Cream et al., 2003, 2004; Plexico et al., 2009; Tichenor \& Yaruss, 2019). Research suggests that confrontation naming data may provide a means for objectively commenting on the mechanisms of word substitution behaviour, including its frequency, variants, time efficiency, and relationship to speaker characteristics (Newman \& Bernstein Ratner, 2007). The present study used a specifically designed picture-naming tool for investigation of verbal avoidance.

The Picture Naming Task (PNT; O’Beirne, 2016) was developed using pictures from Snodgrass and Vanderwart's (1980) standardized 260-item set. This picture set consists of black-and-white line drawings of common everyday objects, normed for name and image agreement, familiarity, and visual complexity. The picture set was originally intended for research examining picture and word processing, with the normative information providing a means for the experimental control of different name and image characteristics. Updated data on name agreement in English have also been published for Snodgrass and Vanderwart's pictures (Yoon et al., 2004). The normative data were obtained from 113 healthy American English-speaking adults, who were asked to provide "the first name of the object that comes to mind" for each picture item (p. 641). Using the collected responses, the authors documented the 'dominant' or 
most common noun provided by participants for each picture, as well as all response variants or alternative names, listed in order of their relative frequencies.

Several studies investigating confrontation naming performance in stuttering speakers have used a subset of Snodgrass and Vanderwart's (1980) pictures, measuring response accuracy against dominant names from the name agreement database (e.g., Anderson, 2008; Maxfield, 2020, 2021; Pellowski, 2011; Pellowski \& Conture, 2005). In the work of Pellowski and Conture, naming responses deviating in any way from the picture's dominant name were considered as errors and excluded from speech reaction latency analyses. Maxfield (2020, 2021), appeared to follow a similar methodology and disregarded alternative word choices (e.g., word substitutions). On the other hand, Anderson accepted as accurate both dominant picture names and close synonyms (e.g., bunny for rabbit), in a study examining semantic and phonological priming effects on picture naming in children with stuttering. It is unclear to what extent Anderson's synonym acceptability judgements were based on published name agreement norms. No prior research has involved the closer inspection of specific word choices associated with the Snodgrass and Vanderwart (1980) data, including both dominant picture names and word alternatives, for the construction of a scaled picture-naming accuracy criterion. This was crucial to development of the PNT tool used in the current study.

A subset of 64 pictures from Snodgrass and Vanderwart's (1980) original set were selected for PNT inclusion, based on (1) picture name variability (Yoon et al., 2004), (2) low visual ambiguity, and (3) low likelihood of naming differences among English dialects, as judged by the researchers. Dominant names for included items contained a range of initial phonemes and clusters, but word frequency and phonological factors were not further controlled due to the exploratory nature of the study. An alphabetized list of dominant and most frequent alternative picture names for the selected items is shown in the Appendix (less frequent alternative names were also considered in PNT scoring, but are not listed). The system of documentation employed by Yoon et al. was thus established as an evidence-based accuracy criterion against which new naming responses could be compared, to determine the usuality (or non-usuality) of different word choices. Assuming PWS will anticipate stuttering during a single-word confrontation task, we hypothesized that stuttering anticipation might be reflected by either unusual word
Table 1

Age and sex characteristics for adult participants with stuttering (PWS) and adult participants with no stuttering (PWNS), and Stuttering Severity Instrument - Fourth Edition (SSI-4; Riley \& Bakker, 2009) overall scores for PWS only

\begin{tabular}{lcccccc}
\hline PWS & Age & Sex & SSI-4 Total Score & PWNS & Age & Sex \\
\hline 1 & 22 & M & 25 & 1 & 25 & M \\
2 & 68 & F & 30 & 2 & 63 & F \\
3 & 60 & F & 11 & 3 & 57 & F \\
4 & 64 & M & 13 & 4 & 62 & M \\
5 & 59 & M & 10 & 5 & 60 & M \\
6 & 62 & F & 9 & 6 & 61 & F \\
7 & 26 & F & 33 & 7 & 22 & F \\
8 & 69 & F & 26 & 8 & 64 & F \\
9 & 27 & F & 34 & 9 & 28 & F \\
Mean & 50.78 & & 21.22 & Mean & 49.11 & \\
SD & 18.53 & & 9.79 & SD & 17.21 & \\
\hline
\end{tabular}

choice and/or delayed word production. Accordingly, the following questions were posed:

(1) Do PWS differ from PWNS on the PNT, in terms of error frequency, response latency, and response accuracy?

(2) Are there significant relationships between response accuracy on the PNT and response latency, error frequency, and participant characteristics such as age and stuttering severity?

\section{Method}

\subsection{Participants}

Participants were nine adult PWS (3 males, 6 females) and nine PWNS matched for sex and age ( \pm 5 years). All participants were native speakers of English, with no reported or observed neurological or speech-language impairment (with the exception of stuttering for PWS). Stuttering was self-reported by each PWS participant, and confirmed by speechlanguage pathologist observation and assessment using the Stuttering Severity Instrument - Fourth Edition (SSI-4; Riley \& Bakker, 2009). All participants provided written consent for their involvement in the study, which was approved by the University of Canterbury Human Ethics Committee (approval number HEC2016/11). Participant characteristics are summarized in Table 1.

\subsection{Procedure}

All procedures and analyses were completed by the first author. Each participant individually completed the PNT in a quiet room. Prior to beginning 

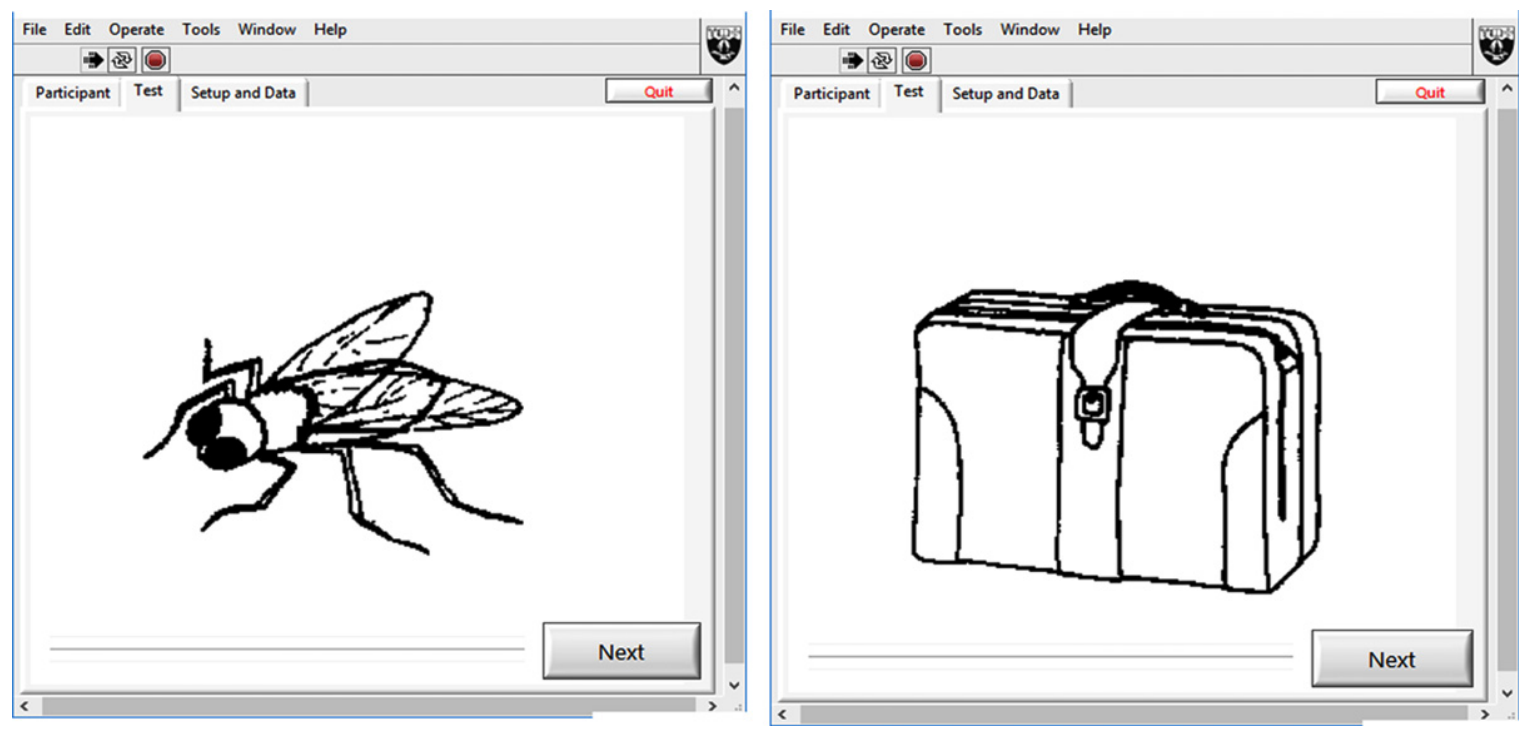

Fig. 1. Screenshot examples from the Picture Naming Task (O’Beirne, 2016). Picture stimuli are from Snodgrass and Vanderwart (1980). The dominant names for these pictures are fly (nondominant name is mosquito) and suitcase (nondominant name is briefcase).

the task, participants were instructed to "name each picture as fast as you can, saying just the name for the picture". PWS participants were additionally encouraged to "use your natural speaking voice, not a speech technique you may have learned in therapy". This was to elicit habitual patterns of speech and language rather than learned behaviours from treatment.

Pictures were presented individually and in random order in the centre of a computer screen. Each picture measured approximately $500 \times 500$ pixels. The appearance of each picture coincided with a simultaneous audible acoustic tone, following which the participant was required to respond. Screen shots illustrating examples of the picture presentation procedure are shown in Fig. 1. Movement from one picture to the next was manually controlled by pressing a key on the computer keyboard. The task began with 10 practice items, consisting of pictures from Snodgrass and Vanderwart's (1980) set that were not included in the actual test. The purpose of the practice period was to familiarize participants with the procedure and to provide feedback regarding performance expectations. Participants were informed when the practical trials had ended and the task was about to begin.

Responses to the 64 test items were automatically audio-recorded by the software using the computer's built-in microphone, and saved as.wav audio files. These were later imported into Praat 6.0 (Boersma \& Weenink, 2015) and displayed as amplitude-by- time waveforms. Vertical cursors were superimposed on each waveform and positioned at the onset of the audible acoustic tone (occurring simultaneous to picture appearance) and the onset of the verbal acoustic signal. The time period between the cursors was taken as the verbal response latency. Responses that were preceded by filled pauses, stuttering moments, comments, or other inappropriate vocalizations, and non-responses, were marked as errors and excluded from response latency analysis. Verbal response latencies that fell more than two standard deviations above or below the mean response latency for each participant were marked as outliers and also excluded (Hennessey et al., 2008). Intra-rater measurement reliability was determined for $10 \%$ of the total audio data. The Pearson product-moment correlation coefficient between the original and repeat measurements for verbal response latency was 0.98 , and the mean absolute difference between the two sets of measures was $0.03 \mathrm{~s}$, indicating a high level of reliability.

All naming responses (including those responses that were judged to have verbal latency errors \& outliers), were analysed for accuracy by comparison to the normative data listed by Yoon et al. (2004). Responses were scored 'match' if they corresponded to the dominant name for a stimulus item, 'other' if they corresponded to any alternative (nondominant) name listed for that item, and 'none' if they represented a word label not listed for that item within the normative database. 


\subsection{Statistical analysis}

Two-tailed paired $t$-tests were used for the overall comparison of performance means between the PWS and PWNS groups on error frequency, response latency, and response accuracy for the PNT. Regression analysis was also conducted to model the effects of different variables on response latency and response accuracy, with $p$-values obtained through likelihood ratio testing.

\section{Results}

\subsection{Group differences}

\subsubsection{PNT error and outlier occurrence}

Across 64 trials, the mean number of response errors (e.g., filled pauses, stuttering moments) for the PWS group was $11.5(S D=7.17)$. The mean number of response latencies judged to be outliers for the PWS group was $2.7(S D=1.03)$. For the PWNS group, a mean of 4.6 errors $(S D=4.03)$ and 2.7 outliers $(S D=1.13)$ were produced. Collectively, the total number of errors and outliers was significantly higher for PWS $(M=14.33, S D=7.33)$ than for PWNS ( $M=7.44, S D=3.86)$ [ $t(8)=2.31, p=0.050]$. All instances of response errors and outliers were excluded from the latency analyses.

\subsubsection{PNT response latency}

The mean verbal response latency for the PWS group was $1.15 \mathrm{~s}(\mathrm{SD}=0.17)$. The mean response latency for the PWNS groups was $1.21 \mathrm{~s}(\mathrm{SD}=0.30)$. Significance testing indicated no difference in mean response latency between the PWS and PWNS group $[t(8)=0.41, p=0.692]$.

\subsubsection{PNT response accuracy}

Across the 64 trials, the mean number of responses produced by the PWS group that were match responses was $41.8(\mathrm{SD}=5.13)$. The mean number of match responses by the PWNS groups was 43.5 ( $\mathrm{SD}=3.92)$. Significance testing revealed no difference between groups in their response accuracy $[t(8)=0.83, p=0.429]$.

\subsection{Regression modelling for response latency}

$\mathrm{R}$ version 3.2.2 ( $\mathrm{R}$ Core Team, 2015) and the lme4 package (Bates et al., 2015) were used to construct a linear mixed effects model for PNT response
Table 2

Linear mixed effects predictors of the Picture Naming Test response latency, with $95 \%$ confidence intervals reported in parentheses

\begin{tabular}{|c|c|c|c|c|}
\hline & $\sigma^{2}$ & $S D$ & & $p$ \\
\hline Item & 0.01 & 0.11 & & $<0.001$ \\
\hline \multirow[t]{2}{*}{ Participant } & 0.02 & 0.13 & & $<0.001$ \\
\hline & $b$ & $S E B$ & $\beta$ & $p$ \\
\hline Intercept & $\begin{array}{c}0.21 \\
(-0.08,0.49)\end{array}$ & 0.14 & & $<0.001$ \\
\hline Age & $\begin{array}{c}-0.00 \\
(-0.01,0.00)\end{array}$ & 0.00 & -0.18 & 0.303 \\
\hline Group: AWS & $\begin{array}{c}-0.55 \\
(-0.95,-0.15)\end{array}$ & 0.19 & -0.90 & 0.644 \\
\hline Accuracy: other & $\begin{array}{c}0.06 \\
(0.02,0.10)\end{array}$ & 0.02 & 0.08 & $<0.001$ \\
\hline Accuracy: none & $\begin{array}{c}0.14 \\
(0.08,0.21)\end{array}$ & 0.03 & 0.12 & $<0.001$ \\
\hline Age x Group: AWS & $\begin{array}{c}0.01 \\
(0.00,0.02)\end{array}$ & 0.00 & 0.97 & 0.010 \\
\hline
\end{tabular}

marginal $R^{2}=0.11 ;$ conditional $R^{2}=0.44$.

latency, in order to identify the relative effects of response accuracy and different participant variables on response latency (Field et al., 2012). Response latency values were first log-transformed to obtain a normal distribution. The mixed effects model was then constructed using a backward stepwise method. The final model contained response accuracy (Accuracy), participant age (Age), and PWS or PWNS group status (Group) as fixed effects, with an Age $\mathrm{x}$ Group interaction term. Intercepts for Item and Participant were entered as random effects, to account for variation related to a particular stimulus item or individual. Visual inspection of residual plots did not reveal any obvious deviations from homoscedasticity or normality. $P$-values for each effect were obtained by likelihood ratio testing of the full model against the model without the effect in question.

Coefficients (with confidence intervals), standard errors, and $p$-values for the fitted model are displayed in Table 2. There was a significant main effect of Accuracy, whereby other responses $(M=1.15 \mathrm{~s}, S D=0.48), F(2,8)=11.31$, $b=0.06, t(8)=2.917, p<0.001$, and none responses $(M=1.35 \mathrm{~s}, \quad S D=0.43), \quad F(2,8)=11.31, \quad b=0.14$, $t(8)=4.255, p<0.001$, were both associated with increased response latency, as compared to match $(M=1.07 \mathrm{~s}, S D=0.42)$ responses. The effect of Accuracy is depicted in Fig. 2.

There was no main effect of Age or Group, indicating that response latency was similar for both the PWS $(M=1.15 \mathrm{~s}, S D=0.17)$ and PWNS groups $(M=1.21 \mathrm{~s}, S D=0.30)$. However, there was a small but significant Age x Group interaction, 


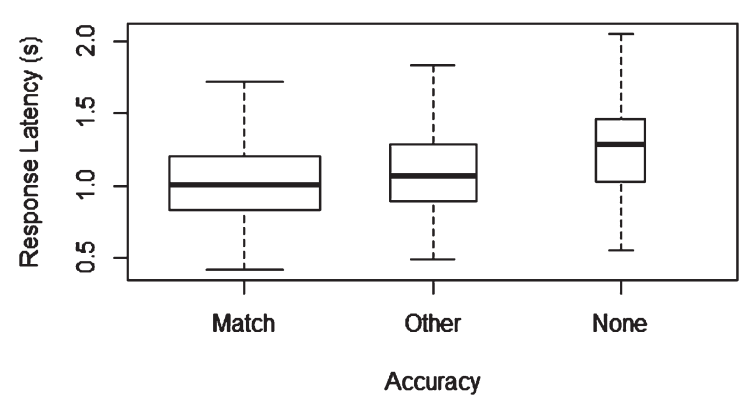

Fig. 2. Boxplot of Picture Naming Task response latency, with box width representing the number of responses scored as match $(n=782)$, other $(n=257)$, and none $(n=113)$, and mean values indicated within the boxes.

$F(1,8)=8.06, b=0.01, t(8)=2.839, p=0.010$, suggesting that increased age was associated with increased response latency for the AWS group only.

\subsection{Regression modelling for response accuracy}

$\mathrm{R}$ version 3.2.2 ( $\mathrm{R}$ Core Team, 2015) and the mlogit package (Croissant, 2013) were used to perform a multinomial logistic regression analysis for PNT response accuracy. A multinomial logistic approach was taken because accuracy was measured as a nominal variable having three possible outcomes (i.e., match, other, none) (Field et al., 2012). The model was constructed using a forward stepwise method. The final model contained only Item and Error (i.e., the production of filled pauses, comments, other inappropriate vocalizations, or moments of stuttering prior to the intended verbal response, and non-responses) as predictors for response accuracy $\left(\mathrm{R}^{2}=0.35\right) . P$-values for the predictors were obtained by likelihood ratio testing of the model in question against the baseline model.

Instances of error/outlier verbal latency production were found to be significantly associated with increased likelihood of none responses, $F(1,8)=16.00, \quad b=1.31, \quad \mathrm{t}(8)=4.00, \quad p<0.001$, $\mathrm{OR}=3.71,95 \% \quad C I[1.95,7.05]$. These were instances where the word label provided by the participant was not listed for a given item within Yoon et al.'s (2004) normative database. No similar relationship was observed for other responses, $\mathrm{F}(1,8)=0.21, b=0.13, \mathrm{t}(8)=0.46, p=0.647$. A tendency towards increased odds of none responses was also observed for certain items on the PNT, but the effect of Item did not reach statistical significance.

PNT response accuracy was then modelled separately for the PWS group using the same method, with
Item and stuttering severity as predictors in the final model $\left(R^{2}=0.43\right)$. More severe stuttering, as measured by SSI-4 total score (Riley \& Bakker, 2009), was found to be significantly associated with slightly reduced odds of both none responses, $F(1,8)=4.67$, $b=-0.04, t(8)=-2.16, p=0.031, \mathrm{OR}=0.96,95 \% C I$ $[0.93,1.00]$, and other responses, $F(1,8)=11.49$, $b=-0.04, t(8)=-3.39, p<0.001, \mathrm{OR}=0.96,95 \% C I$ $[0.93,0.98]$. Again, the effect of Item did not reach statistical significance.

\section{Discussion}

People who stutter are able to anticipate a moment of stuttering (Bloodstein et al., 2021). The present study attempted to capture stuttering anticipation on the basis of word choice. We hypothesized that stuttering anticipation might be reflected by either unusual word choice and/or delayed word production during a single-word confrontation naming task. The results showed that the PWS group produced significantly more total errors and outliers (indicative of word-finding difficulty, hesitation, or disfluency) than the PWNS group. Notwithstanding methodological variation, this corresponds to Newman and Bernstein Ratner's (2007) observation that PWS participants differed from fluent controls on number of errors, but not response variants, during rapid picture naming. Past studies that have included name response variants within total error counts have reported a comparable error frequency between the two speaker groups (Hennessey et al., 2008; Maxfield et al., 2010, 2015). Therefore, the present findings support previous research suggesting a higher incidence of word finding and fluency errors among PWS than PWNS. These data reflect the presence of more frequent disfluent moments in the speech of PWS, whether in the form of stutter-like or typical disfluencies, and their capacity to interfere with rapid verbal responding.

The two key performance variables in this study were the accuracy of naming responses on the PNT, assessed against Yoon et al.'s (2004) normative data using a three-way criterion, and the response latencies associated with these responses. Overall, the PWS and PWNS groups did not differ significantly on naming accuracy or response latency. The task yielded similar data to a number of existing reports in the literature (Crowe \& Kroll, 1991; Hennessey et al., 2008; Maxfield et al., 2010, 2015; Maxfield, 2020, 2021; Newman \& Bernstein Ratner, 2007; Prins et al., 1997), in spite of the novel accuracy criterion 
proposed as a means of capturing the usuality (or non-usuality) of word choices. The PWS participants were no more likely than control participants to produce other or none responses, as might have been expected in the case of frequent word substitution. It is known that use of verbal avoidance behaviours by PWS varies according to speaking situation (Crichton-Smith, 2002; Plexico et al., 2009); the PNT involved single word production in a research setting. Such a contextual environment may have reduced any perceived threat of emotional harm from stuttering, and thus minimized habitual reliance on word substitution and related strategies of the PWS participants. Alternatively, it is possible that individual avoidance patterns, being highly idiosyncratic, were not reflected in systematic differences at the level of group analyses.

Despite the lack of PNT sensitivity to particular aspects of confrontation naming behaviour in PWS, statistical modelling of task accuracy and latency measures revealed several interesting relationships. When verbal responding on the PNT was preceded by an error (e.g., interjection, filled pause), both PWS and PWNS participants were almost four times more likely to label picture stimuli using non-usual words not found in Yoon et al.'s (2004) normative database. With item and participant variability accounted for, and excluding errors, none responses were also associated with a longer response latency than responses matching the dominant names documented by Yoon et al. A similar latency difference was observed for other responses representing non-dominant name variants. Considering as 'substitution' any variation in naming behaviour from the normative criterion (i.e., all instances of other and none responses), the data may therefore be summarized as follows: in both PWNS and PWS speakers, word substitution is (1) more likely to occur, in its most non-usual forms, subsequent to obvious errors of word finding or fluency, and (2) associated with a certain production time-cost. While current PNT methodology does not allow for the disentangling of the roles of lexical processing, speech fluency, and their mutual effects, the behavioural observations suggest a certain generality of word substitution as a verbal action. Switching words in the moment might provide a means of avoiding the emotional stresses not only of established stuttering, but, more generally, of any systemic difficulty hindering verbal expression. The present data also provide objective evidence of a production delay associated with word substitution, corroborating PWS self-reports regarding the additional processing demands of using such strategies (Cream et al., 2003, 2004; Plexico et al., 2009).

Further to the relationships already described, a significant effect of participant age on PNT response latency was also observed, but only for the AWS group. Participant groups in this study were age- and sex-matched, with individuals ranging in age from 22 to 69 years. Changes in brain function for speech and language production are known to occur with healthy aging (e.g., Madhavan et al., 2014; Tremblay \& Deschamps, 2016). These are accompanied by declines in the speed and accuracy of verbal performance, which become more pronounced as task complexity increases (e.g., Ashaie \& Obler, 2014; Fletcher et al., 2015; Tremblay \& Deschamps, 2016). The age-by-group interaction observed in the current study suggests a special vulnerability among PWS to the changing demands-capacities balance brought about by aging, whereby an age effect on response latency emerges at a lower complexity threshold. Such a notion is not new; various studies have shown that complexity factors such as word frequency, sentence complexity, and emotionality, all exert a relatively greater burden on verbal responding capacity in AWS than AWNS (Hennessey et al., 2008, 2014; Newman \& Bernstein Ratner, 2007; Tsiamtsiouris \& Cairns, 2009). These data support the classic demands and capacities model of stuttering (Starkweather, 1987; Starkweather \& Gottwald, 1990), and bring to light the magnified cost for PWS of any added source of communication stress, such as that implied in active verbal avoidance.

Lastly, an unexpected inverse relationship was observed between stuttering severity and frequency of other and none responses for the PWS group. Participants with higher total scores on the SSI-4 (Riley \& Bakker, 2009) were slightly less likely to produce non-usual words in response to the picture stimuli. The negative directionality of the relationship is counter-intuitive, possibly reflecting a certain reduction in lexical creativity or freedom as a conditioned fear response to severe stuttering. Similar restrictions in the conversational expression of PWS have been shown using functional linguistic data (Lee et al., 2015, 2016b). This result can also be interpreted in light of research examining generalized and social anxiety in PWS. Studies in this area have failed to find a straightforward positive correlation between stuttering severity and anxiety (of which verbal avoidance is a manifestation), as measured using various physiological and self-report indices (Blumgart et al., 2010; Craig et al., 2003; Ezrati-Vinacour 
\& Levin, 2004; Mulcahy et al., 2008). The emotional harm of stuttering may also persist even in the face of apparent post-treatment speech fluency (Cream et al., 2004). These observations demonstrate the complexity of the relationship between overt stuttering and its covert features, which ultimately intensifies the need for objective anxiety and avoidance data to more effectively manage the impact of stuttering.

\subsection{Future directions and conclusion}

We emphasize that the present study was exploratory in nature and design. In addition to study replication with a larger sample size, further development of the PNT would improve its sensitivity to target behaviours and its utility in this area of investigation. There is a clear role of linguistic complexity in moderating fine aspects of picture-naming performance in PWS (Hennessey et al., 2008, 2014; Newman \& Bernstein Ratner, 2007; Tsiamtsiouris \& Cairns, 2009). Current PNT stimuli consisted of pictured nouns only, and the word frequency, familiarity, and phonological features of the items selected from Snodgrass and Vanderwart (1980) were not carefully controlled. Expansion of the stimulus set to include verbs, which represent a less simple word class than nouns (Bloodstein et al., 2021; Maxfield, 2021), would introduce an added complexity element. This would reveal subtler differences in word choice and naming behaviour between PWS and PWNS (Prins et al., 1997). Similarly, increasing experimental control of stimulus characteristics using the wider range of normative data published by Snodgrass and Vanderwart would minimize any confounding item effects. Item effects in the current study, though not statistically significant, explained a certain proportion of the variation in response accuracy and latency. While this variation was to some extent accounted for through the use of mixed effects modelling, further standardization of task stimuli would enable stutteringrelated word choices to be discerned with greater clarity.

To conclude, use of the PNT has provided evidence of (1) a time-cost associated with word substitution, (2) the effects of processing demands on speech-language performance in PWS, and (3) the independence of stuttering impact from severity of actual speech disfluency. These results confirm the need for a greater understanding of verbal avoidance behaviour in AWS, based on objective sources of evidence. The PNT paradigm, with its focus on word choice in the context of timed responding, provides an original methodological model for exploring the mechanisms and demands of word substitution in these speakers. The current version of the task did not, however, permit the specific evaluation of word substitution behaviour in PWS, in terms of response variant and latency characteristics within a confrontation naming context. Continued research and task development will enlighten aspects of verbal avoidance central to the effective management of stuttering impact.

\section{Acknowledgments}

The authors acknowledge the individuals who participated in this study.

\section{Conflict of interest}

The authors report no conflict of interest. Given his role as an Editorial Board Member, Michael P. Robb had no involvement nor access to information regarding the peer review of this article.

\section{References}

Amster, B. \& Klein, E. (2018). More than fluency: The social emotional, and cognitive dimensions of stuttering. Plural Publishing.

Anderson, J.D. (2008). Age of acquisition and repetition priming effects on picture naming of children who do and do not stutter. Journal of Fluency Disorders, 33, 135-155. https://psycnet.apa.org/doi/10.1016/j.jfludis.2008.04.001

Ashaie, S., \& Obler, L. (2014). Effect of age, education, and bilingualism on confrontation naming in older illiterate and low-educated populations. Behavioural Neurology, Article ID 970520. https://doi.org/10.1155/2014/970520

Bates, D., Maechler, M., Bolker, B., \& Walker, S. (2015). Fitting linear mixed-effects models using lme4. Journal of Statistical Software, 67, 1-48. https://doi.org/10.18637/jss.v067.i01

Blomgren, M., Roy, N., Callister, T., \& Merrill, R.M. (2005). Intensive stuttering modification therapy: A multidimensional assessment of treatment outcomes. Journal of Speech, Language, and Hearing Research, 48, 509-523. https://doi.org/10.1044/1092-4388(2005/035)

Bloodstein, O., Bernstein Ratner, N. \& Brundage, S. (2021). A handbook on stuttering ( $7^{\text {th }}$ ed.). Plural Publishing.

Blumgart, E., Tran, Y., \& Craig, A. (2010). Social anxiety disorder in adults who stutter. Depression and Anxiety, 27, 687-692. DOI: $10.1002 /$ da. 20657

Boersma, P. \& Weenink, D. (2015). Praat 6.0. [Computer software]. Amsterdam: University of Amsterdam.

Briley, P., \& Kalinowski, J. (2016). General vulnerability to stuttering: The experience of stuttering and conditions bringing 
about invulnerability. Medical Hypotheses, 93, 55-61. DOI: 10.1016/j.mehy.2016.05.016

Craig, A., Hancock, K., Tran, Y., \& Craig, M. (2003). Anxiety levels in people who stutter: A randomized population study. Journal of Speech, Language, and Hearing Research, 46, 1197 1206. https://doi.org/10.1044/1092-4388(2003/093)

Cream, A., Onslow, M., Packman, A., \& Llewellyn, G. (2003). Protection from harm: The experience adults after therapy with prolonged-speech. International Journal of Language and Communication Disorders, 38, 379-395. DOI: 10.1080/13682820310001598166

Cream, A., Packman, A., \& Llewellyn, G. (2004). The playground rocker: A metaphor for communication after treatment for adults who stutter. Advances in Speech-Language Pathology, 6, 182-187. https://doi.org/10.1080/14417040412331283048

Crichton-Smith, I. (2002). Communicating in the real world: Accounts from people who stammer. Journal of Fluency Disorders, 27, 333-352. DOI: 10.1016/s0094-730x(02)00161-4

Croissant, Y. (2013). mlogit: multinomial logit model. R package version 0.2-4. http://CRAN.R-project.org/package=mlogit.

Crowe, K. M., \& Kroll, R. M. (1991). Response latency and response class for stutterers and nonstutterers as measured by a word-association task. Journal of Fluency Disorders, 16, 35-54. https://doi.org/10.1016/0094-730X(91)90034-A

Ezrati-Vinacour, R., \& Levin, I. (2004). The relationship between anxiety and stuttering: A multidimensional approach. Journal of Fluency Disorders, 29, 135-148. DOI: 10.1016/j.jfludis.2004.02.003

Field, A., Miles, J., \& Field, Z. (2012). Discovering statistics using $R$. Sage Publishing.

Fletcher, A.R., McAuliffe, M.J., Lansford, K.L., \& Liss, J.M. (2015). The relationship between speech segment duration and vowel centralization in a group of older speakers. Journal of the Acoustical Society of America, 138, 2132-2139. DOI: 10.1121/1.4930563

Folstein, J.R., \& Van Petten, C. (2008). Influence of cognitive control and mismatch on the N2 component of the ERP: A review. Psychophysiology, 45, 152-170. DOI: 10.1111/j.14698986.2007.00602.x

Garcia-Barera, M., \& Davidow, J. (2015). Anticipation in stuttering: A theoretical model of the nature of stutter prediction. Journal of Fluency Disorders, 44, 1-15. DOI: 10.1016/j.jfludis.2015.03.002

Guitar, B. (2019). Stuttering: An integrated approach to its nature and treatment (5th ed.). Lippincott Williams \& Wilkins.

Hennessey, N.W., Dourado, E., \& Beilby, J.M. (2014). Anxiety and speaking in people who stutter: An investigation using the emotional Stroop task. Journal of Fluency Disorders, 40, 44-57. DOI: 10.1016/j.jfludis.2013.11.001

Hennessey, N. W., Nang, C. Y., \& Beilby, J. M. (2008). Speeded verbal responding in adults who stutter: Are there deficits in linguistic encoding? Journal of Fluency Disorders, 33, 180202. DOI: $10.1016 /$ j.jfludis.2008.06.001

Iverach, L., Menzies, R.G., O’Brian, S., Packman, A., \& Onslow, M. (2011). Anxiety and stuttering: Continuing to explore a complex relationship. American Journal of SpeechLanguage Pathology, 20, 221-232. https://doi.org/10.1044/ 1058-0360(2011/10-0091)

Jackson, E.S., Yaruss, J.S., Quesal, R.W., Terranova, V., \& Whalen, D.H. (2015). Responses of adults who stutter to the anticipation of stuttering. Journal of Fluency Disorders, 45, 38-51. DOI: 10.1016/j.jfludis.2015.05.002
Lee, A., van Dulm, O., Robb, M.P., \& Ormond, T. (2015). Communication restriction in adults who stutter. Clinical Linguistics \& Phonetics, 29, 536-556. https://doi.org/10.3109/ 02699206.2015.1030039

Lee, A., Robb, M.P., van Dulm, O., \& Ormond, T. (2016a). Communication restriction in adults who stutter: Part II. Clinical Linguistics \& Phonetics, 30, 546-567. https://doi.org/10.3109/02699206.2016.1151939

Lee, A., Robb, M.P., van Dulm, O., \& Ormond, T. (2016b). Communication restriction in adults who stutter: Part III. Clinical Linguistics \& Phonetics, 30, 911-924. DOI: 10.1080/02699206.2016.1206622

Levelt, W. J., Roelofs, A., \& Meyer, A. S. (1999). A theory of lexical access in speech production. Behavioral and Brain Sciences, 22, 1-38. DOI: 10.1017/s0140525x99001776

Linklater, J. (2020). Principles of avoidance-reduction therapy. In T. Steward (Ed.), Stammering resources for adults and teenagers: Integrating new evidence into clinical practice (pp.110-141). Routledge. DOI: 10.4324/9780429263 835-5

Lowe, R., Helgadottir, F., Menzies, R., Heard, R., O’Brian, S., Packman, A., \& Onslow, M. (2017). Safety behaviors and stuttering. Journal of Speech, Language, and Hearing Research, 60, 1246-1253. https://doi.org/10.1044/2016_JSLHR-S-160055

Lowe, R., Menzies, R., Onslow, M., Packman, A., \& O’Brian, S. (2021). Speech and anxiety management with persistent stuttering: Current status and essential research. Journal of Speech, Language, and Hearing Research, 64, 59-74. https://doi.org/10.1044/2020_JSLHR-20-00144

Madhavan, K.M., McQueeny, T., Hower, S.R., Shear, P., \& Szaflarski, J. (2014). Superior longitudinal fasciculus and language functioning in healthy aging. Brain Research, 1562, 11-22. DOI: 10.1016/j.brainres.2014.03.012

Mancinelli, J. (2018). A perspective on stuttering in the social context. In B. Amster, \& E. Klein (Eds.), More than fluency: The social emotional, and cognitive dimensions of stuttering (pp. 45-68). Plural Publishing.

Maxfield, D.N. (2021). Cognitive control of action naming in adults who stutter. Journal of Fluency Disorders, 70, 1-17. DOI: $10.1016 /$ j.jfludis.2021.105841

Maxfield, D.N. (2020). Inhibitory control of lexical selection in adults who stutter. Journal of Fluency Disorders, 66, 1-19. https://doi.org/10.1016/j.jfludis.2020.105780

Maxfield, D.N., Huffman, J.L., Frisch, S.A., \& Hinckley, J.J. (2010). Neural correlates of semantic activation spreading on the path to picture naming in adults who stutter. Clinical Neurophysiology, 121, 1447-1463. DOI: 10.1016/j.clinph.2010.03.026

Maxfield, D.N., Morris, K., Frisch, S.A., Morphew, K., \& Constantine, J.L. (2015). Real-time processing in picture naming in adults who stutter: ERP evidence. Clinical Neurophysiology, 126, 284-296. DOI: 10.1016/j.clinph.2014.05.009

Miyake, A., Friedman, N. P., Emerson, M. J., Witzki, A. H., Howerter, A., \& Wager, T. D. (2000). The unity and diversity of executive functions and their contributions to complex "frontal lobe" tasks: A latent variable analysis. Cognitive Psychology, 41, 49-100. DOI: 10.1006/cogp.1999.0734

Mulcahy, K., Hennessey, N., Beilby, J., \& Byrnes, M. (2008). Social anxiety and the severity and typography of stuttering in adolescents. Journal of Fluency Disorders, 33, 306-319. DOI: 10.1016/j.jfludis.2008.12.002 
Newman, R., \& Bernstein Ratner, N. (2007). The role of selected lexical factors on confrontation naming accuracy, speed, and fluency in adults who do and do not stutter. Journal of Speech, Language, and Hearing Research, 50, 196-213. DOI: 10.1044/1092-4388(2007/016)

O'Beirne, G. (2016). Picture naming task. [Computer software]. Christchurch: University of Canterbury.

Pellowski, M.W. (2011). Word-finding and vocabulary abilities of adults who do and do not stutter. Contemporary Issues in Communication Science and Disorders, 38, 126-134. https://doi.org/10.1044/cicsd_38_F_126

Pellowski, M.W., \& Conture, E.G. (2005). Lexical priming in picture naming of young children who do and do not stutter. Journal of Speech, Language, and Hearing Research, 48, 278-294. DOI: 10.1044/1092-4388(2005/019)

Petrunik, M., \& Shearing, C. (1983). Fragile facades: Stuttering and the strategic manipulation of awareness. Social Problems, 31, 125-138. https://doi.org/10.1525/sp.1983.31.2.03a00010

Plexico, L.W., Manning, W.H., \& Levitt, H. (2009). Coping responses by adults who stutter: Part I. Protecting the self and others. Journal of Fluency Disorders, 34, 87-107. DOI: 10.1016/j.jfludis.2009.06.001

Prins, D., Main V., \& Wampler, S. (1997). Lexicalization in adults who stutter. Journal of Speech, Language, and Hearing Research, 40, 373-384. DOI: 10.1044/jslhr.4002.373

$\mathrm{R}$ Core Team (2015). $R$ : A language and environment for statistical computing. R Foundation for Statistical Computing, Vienna, Austria. URL http://www.R-project.org/.

Riley, G.D., \& Bakker, K. (2009). Stuttering severity instrument: SSI-4. Pro-Ed.

Shao, Z., Roelofs, A., Acheson, D.J., \& Meyer, A.S. (2014). Electrophysiological evidence that inhibition supports lexical selection in picture naming. Brain Research, 1586, 130-142. https://doi.org/10.1016/j.brainres.2014.07.009

Shao, Z., Roelofs, A., \& Meyer, A.S. (2012). Sources of individual differences in the speed of naming objects and actions: The contribution of executive control. The Quarterly Journal of Experimental Psychology, 65, 1927-1944. https://doi.org/10.1080/17470218.2012.670252

Snodgrass, J.G., \& Vanderwart, M. (1980). A standardized set of 260 pictures: Norms for name agreement, image agreement, familiarity, and visual complexity. Journal of Experimental Psychology: Human Learning and Memory, 6, 174-215. https://doi.org/10.1037/0278-7393.6.2.174
Starkweather, C.W. (1987). Fluency and stuttering. Prentice-Hall, Inc.

Starkweather, C.W., \& Gottwald, S.R. (1990). The Demands and Capacities Model II: Clinical applications. Journal of Fluency Disorders, 15, 143-157. https://doi.org/10.1016/0094730X(90)90015-K

Taylor, W. L., Lore, J. I., \& Waldman, I. N. (1970). Latencies of semantic aphasics, stutterers, and normal controls to cloze items requiring unique and non-unique oral responses. In Proceedings of the Annual Convention of the American Psychological Association, 78, 75-76, Washington, DC: American Psychological Association.

Tichenor, S. \& Yaruss, J.S. (2019). Stuttering as defined by adults who stutter. Journal of Speech, Language, and Hearing Research, 62, 4356-4369. https://doi.org/10.1044/2019_ JSLHR-19-00137

Tremblay, P., \& Deschamps, I. (2016). Structural brain aging and speech production: A surface-based brain morphometry study. Brain Structure \& Function, 221, 3275-3299. DOI: 10.1007/s00429-015-1100-1

Tsiamtsiouris, J., \& Cairns, H.S. (2009). Effects of syntactic complexity and sentence-structure priming on speech initiation times in adults who stutter. Journal of Speech, Language, and Hearing Research, 52, 1623-1639. https://doi.org/10.1044/1092-4388(2009/08-0063)

Vanryckeghem, M., Brutten, G.J., Uddin, N., \& Van Borsel, J. (2004). A comparative investigation of the speech-associated coping responses reported by adults who do and do not stutter. Journal of Fluency Disorders, 29, 237-250. DOI: 10.1016/j.jfludis.2004.07.001

Yoon, C., Feinberg, F., Luo, T., Hedden, T., Gutchess, A.H., Chen, H.M., Mikels, J.A., Jiao, S., \& Park, D.C. (2004). A cross-culturally standardized set of pictures for younger and older adults: American and Chinese norms for name agreement, concept agreement, and familiarity. Behavior Research Methods, Instruments, \& Computers, 36, 639-649. DOI: $10.3758 / \mathrm{bf} 03206545$ 


\section{Appendix}

Alphabetized list of dominant names (with most common non-dominant alternatives in parentheses) for picture stimuli used in the Picture Naming Task (PNT). The PNT used pictures from Snodgrass and Vanderwart (1980), with name agreement data published by Yoon et al. (2004).

1. alligator (crocodile)

2. arrow (left arrow)

3. barn (farm)

4. bear (polar bear)

5. beetle (bug)

6. bird (finch)

7. bottle (wine bottle)

8. bowl (mixing bowl)

9. bread (loaf of bread)

10. cake (pie)

11. car (Oldsmobile)

12. chicken (rooster)

13. cigar (cigarette)

14. couch (sofa)

15. cup (tea cup)

16. doll (girl)

17. doorknob (door handle)

18. dresser (drawer)

19. envelope (letter)

20. finger (index finger)

21. fly (mosquito)

22. French horn (horn)

23. glass (cup)

24. goat (donkey)

25. grasshopper (cricket)

26. hair (ladies hair)

27. hat (top hat)

28. ironing board (iron board)
29. kettle (tea kettle)

30. leaf (maple leaf)

31. lemon (lime)

32. lettuce (cabbage)

33. light switch (switch)

34. lock (padlock)

35. moon (crescent moon)

36. mouse (rat)

37. necklace (pearl necklace)

38. nut (bolt)

39. paintbrush (brush)

40. peach (nectarine)

41. piano (grand piano)

42. pliers (wrench)

43. purse (bag)

44. rabbit (bunny)

45. refrigerator (fridge)

46. rocking chair (chair)

47. rolling pin (roller)

48. sailboat (boat)

49. screw (nail)

50. sheep (lamb)

51. skirt (dress)

52. spinning wheel (wool spinner)

53. stool (chair)

54. suitcase (briefcase)

55. swan (goose)

56. tie (necktie)

57. top (spinning top)

58. truck (semi-truck)

59. TV (television)

60. wagon (kids wagon)

61. watermelon (watermelon slice)

62. wheel (wagon wheel)

63. wineglass (glass)

64. wrench (nut wrench) 